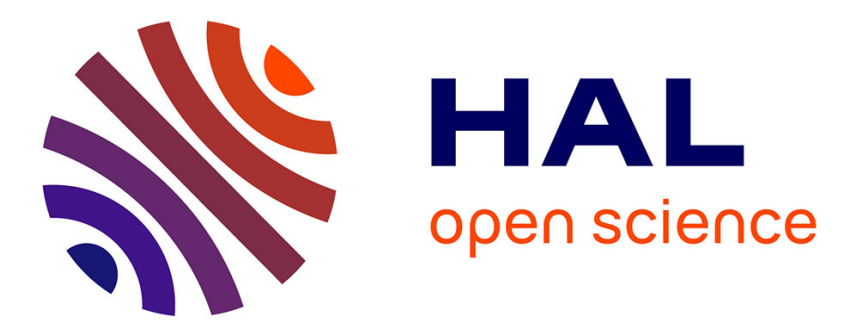

\title{
Nature of magnetocrystalline anisotropy in the basal plane of iron borate
}

Mark Strugatsky, Kira Seleznyova, Sergey Yagupov, Alexey Drovosekov, Janis Kliava

\section{- To cite this version:}

Mark Strugatsky, Kira Seleznyova, Sergey Yagupov, Alexey Drovosekov, Janis Kliava. Nature of magnetocrystalline anisotropy in the basal plane of iron borate. Journal of Magnetism and Magnetic Materials, 2017, 442, pp.417-422. 10.1016/j.jmmm.2017.06.132 . hal-01578819

\section{HAL Id: hal-01578819 https://hal.science/hal-01578819}

Submitted on 29 Aug 2017

HAL is a multi-disciplinary open access archive for the deposit and dissemination of scientific research documents, whether they are published or not. The documents may come from teaching and research institutions in France or abroad, or from public or private research centers.
L'archive ouverte pluridisciplinaire HAL, est destinée au dépôt et à la diffusion de documents scientifiques de niveau recherche, publiés ou non, émanant des établissements d'enseignement et de recherche français ou étrangers, des laboratoires publics ou privés.

\section{다(1)(2)}

Distributed under a Creative Commons Attribution - ShareAlikel 4.0 International 


\title{
Nature of magnetocrystalline anisotropy in the basal plane of iron borate
}

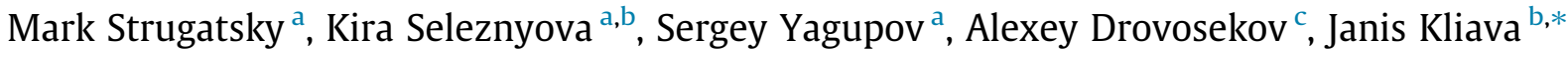 \\ ${ }^{a}$ Physics and Technology Institute, V.I. Vernadsky Crimean Federal University, 4 Vernadsky Avenue, Simferopol 295007, Russia \\ b LOMA, UMR 5798 Université de Bordeaux-CNRS, 33405 Talence cedex, France \\ ${ }^{c}$ P.L. Kapitza Institute for Physical Problems RAS, 2 ul. Kosygina, Moscow 119334, Russia
}

\begin{abstract}
A B S T R A C T
Basal (hexagonal) magnetocrystalline anisotropy of iron borate $\mathrm{FeBO}_{3}$, including crystal field and dipole dipole contributions, is discussed in detail. Previously, the latter contribution had been ruled out on the basis of symmetry; indeed, considering the magnetic dipole dipole interaction in the approximation of point dipoles, only uniaxial magnetocrystalline anisotropy is accounted for. In the present work we con sider the dipole dipole interaction energy for extended dipoles and calculate the dipole dipole contribu tion to basal magnetocrystalline anisotropy for two different models of an extended dipole: a pair of fictitious magnetic charges and a circular current loop. A comparison between theoretical expressions developed and experimental results obtained by antiferromagnetic resonance allows estimating the effective size of magnetic dipoles and calculating the dipole dipole contribution to basal magnetocrys talline anisotropy constants of iron borate.
\end{abstract}

Keywords:

Iron borate

Magnetocrystalline anisotropy

Crystal field

Dipole-dipole interaction

\section{Introduction}

From the standpoint of magnetic properties, iron borate $\mathrm{FeBO}_{3}$ is an easy plane antiferromagnet, possessing a weak in plane fer romagnetic moment. Iron borate has rhombohedral calcite struc ture of point group symmetry $\mathrm{D}_{3 \mathrm{~d}}$ and the space group $\mathrm{D}_{3 \mathrm{~d}}^{6}[1]$. The effective basal (hexagonal) magnetocrystalline anisotropy energy for $\mathrm{FeBO}_{3}$ can be expressed as follows [2]:

$$
\mathcal{E}_{\mathrm{A}} \quad e_{\mathrm{eff}} \cos 6 \varphi
$$

where $\varphi$ is the azimuthal angle of the antiferromagnetic vector and $e_{\text {eff }}$ is the effective basal anisotropy constant,

$e_{\text {eff }} \quad e_{\mathrm{FeBO}_{3}}+\frac{1}{4} \frac{d_{\mathrm{FeBO}_{3}}^{2}}{a_{\mathrm{eff}}}$,

$d_{\mathrm{FeBO}_{3}}$ and $e_{\mathrm{FeBO}_{3}}$ being the basal magnetocrystalline anisotropy con stants and $a_{\text {eff }}$ being the effective uniaxial magnetocrystalline aniso tropy constant,

$a_{\text {eff }} a_{\mathrm{FeBO}_{3}}+\frac{D_{\mathrm{FeBO}_{3}}^{2}}{E_{\mathrm{FeBO}_{3}}}$.

\footnotetext{
* Corresponding author.

E-mail address: janis.kliava@u-bordeaux.fr (J. Kliava).
}

In the latter equation $a_{\mathrm{FeBO}_{3}}, D_{\mathrm{FeBO}_{3}}$ and $E_{\mathrm{FeBO}_{3}}$ are, respectively, the uniaxial magnetocrystalline anisotropy, Dzyaloshinskii Moriya and exchange constants for $\mathrm{FeBO}_{3}$.

As far as for $\mathrm{Fe}^{3+}$ ( $3 d^{5}$ electronic configuration) the orbital moment equals zero, the exchange energy in a good approximation is isotropic [3], so that the magnetocrystalline anisotropy energy of $\mathrm{FeBO}_{3}$ includes only crystal field (cf) and dipole dipole (dip) terms:

$$
\begin{array}{cc}
a_{\mathrm{FeBO}_{3}} & a_{\mathrm{cf}}+a_{\mathrm{dip}} \\
d_{\mathrm{FeBO}_{3}} & d_{\mathrm{cf}}+d_{\mathrm{dip}} \\
e_{\mathrm{FeBO}_{3}} & e_{\mathrm{cf}}+e_{\mathrm{dip}}
\end{array}
$$

The crystal field contributions to the abovementioned constants have been calculated by Seleznev in the mean field approximation [4]. Indeed, the effective exchange field $H_{\mathrm{E}}$ in iron borate is in order of $10^{3} \mathrm{kOe}$, thus, $\mathrm{Fe}^{3+}$ ions experience strong exchange coupling [5]. The crystal field contribution to the anisotropic part of the magne tocrystalline anisotropy energy can be calculated in perturbation theory using the spin Hamiltonian for isolated $\mathrm{Fe}^{3+}$ ion in a dia magnetic crystal isomorphous to iron borate, e.g., gallium borate $[6,7]$. Thus, $a_{\mathrm{cf}}, d_{\mathrm{cf}}$ and $e_{\mathrm{cf}}$ can be expressed through the parame ters of the spin Hamiltonian. The corresponding expressions will be given and the parameters will be specified in the next Section.

The dipole dipole contributions to these constants are usually calculated using the lattice sum method. The value of $a_{\text {dip }} \quad 3.8210^{5} \mathrm{Jm}^{3}$ at $0 \mathrm{~K}$ for $\mathrm{FeBO}_{3}$ has been obtained previously [8]. $a_{\mathrm{FeBO}}$ has been determined by antiferromagnetic resonance 
(AFMR) in a wide temperature range by Velikov et al. [9]; its value extrapolated to $0 \mathrm{~K}$ is $a_{\mathrm{FeBO}_{3}}^{\exp } \quad 3.2910^{5} \mathrm{Jm}^{3}$.

Earlier, the occurrence of the dipole dipole contribution to hexagonal basal anisotropy in iron borate had been ruled out on the grounds of symmetry. Indeed, the dipole dipole interaction energy is usually considered for "point dipoles" having a negligible size, in which case only the uniaxial anisotropy is accounted for. Meanwhile, more sophisticated anisotropies, in particular, the hexagonal magnetocrystalline anisotropy can be described by higher than first order terms in the expansion of the dipole dipole interaction energy in a Taylor series in the small parameter dipole size/interdipole distance. Thus, taking into consideration "extended dipoles", having non negligible size, opens the possibil ity to reasonably account for the dipole dipole contribution to the basal anisotropy constants and, subsequently, to estimate effective dipole dimensions in iron borate.

With this aim in mind, we have developed a theoretical descrip tion of two models of the extended magnetic dipole: (1) an assem bly of two fictitious "magnetic charges" $\pm q$ a distance $d$ apart and (2) an Ampérian current, i.e., a circular current loop of a radius $R$ delimiting an area $S \quad \pi R^{2}$. Previously, we have obtained and dis cussed the expressions of vector potentials and magnetic fields produced by these two models [10]. The purpose of the present work has been to calculate the dipole dipole contribution to the basal magnetocrystalline anisotropy constants of iron borate and to evaluate the size of dipoles in $\mathrm{FeBO}_{3}$ in the framework of the suggested models.

\section{Crystal field contribution to magnetocrystalline anisotropy}

In order to calculate the crystal field contribution we have to consider a $\mathrm{Fe}^{3+}$ ion in a diamagnetic crystal isomorphous with $\mathrm{FeBO}_{3}$. The conventional spin Hamiltonian in this case is $[11,12]$ :

$$
\begin{array}{rl}
\mathcal{H} & g \beta \boldsymbol{H} \cdot \boldsymbol{S}+\frac{1}{3} D O_{2}^{0} \quad \frac{1}{180}\left(\begin{array}{ll}
a & F
\end{array}\right) \mathrm{O}_{4}^{0}+\frac{\sqrt{ } 2}{9} a\left(\mp \mathrm{O}_{4}^{3} \sin 3 \alpha\right. \\
& \left.+\mathrm{O}_{4}{ }^{3} \cos 3 \alpha\right)
\end{array}
$$

where $g$ is close to the free electron $g$ factor $g_{e} \quad 2.0023, \beta$ is the Bohr magneton, $\boldsymbol{H}$ is the magnetizing field, $S \quad \frac{5}{2}$ is the electron spin of $\mathrm{Fe}^{3+}, D$ is the second order axial fine structure constant, $a$ and $F$ are, respectively, the fourth order cubic and axial fine structure constants and $\mathrm{O}_{2}^{0}, \mathrm{O}_{4}^{0}, \mathrm{O}_{4}^{3}$ and $\mathrm{O}_{4}{ }^{3}$ are extended Stevens operators, as defined in the textbook by Al'tshuler and Kozyrev [13]. The $\mp$ signs refer to two non equivalent iron sites with local magnetic axes rotated through the angle $\mp \alpha$ about the $C_{3}$ axis, see Fig. 1 .

The spin Hamiltonian parameters of $\mathrm{Fe}^{3+}$ in diamagnetic $\mathrm{GaBO}_{3}$ have been determined previously by Lukin et al. [11] and recently specified by Seleznyova et al. [12].

Seleznev has obtained the expressions for $a_{\mathrm{cf}}, d_{\mathrm{cf}}$ and $e_{\mathrm{cf}}$ using the mean field approximation [4]:

$$
\begin{array}{ll}
a_{\mathrm{cf}} & 2 N\left[D t(x) \quad \frac{1}{2}\left(\begin{array}{ll}
a & F) r(x)
\end{array}\right]\right. \\
d_{\mathrm{cf}} & \frac{\sqrt{ } 2}{3} N \operatorname{ar}(x) \cos 3 \alpha \\
e_{\mathrm{cf}} & 0
\end{array}
$$

where $N$ is the number of $\mathrm{Fe}^{3+}$ ions per unit volume, $N \quad 2.23610^{28} \mathrm{~m}^{3}$ for $\mathrm{FeBO}_{3}$ and $t(x)$ and $r(x)$ are the following functions:

$$
\begin{array}{ll}
t(x) & \left.\frac{\left(8 \operatorname{ch}_{2}^{1} x+2 \operatorname{ch}_{2}^{3} x\right.}{3} 10 \operatorname{ch}_{2}^{5} x\right) \operatorname{sh} \frac{1}{2} x \\
\text { and } & \\
r(x) & 5 \frac{\left(2 \operatorname{ch}_{2}^{1} x \quad 3 \operatorname{ch}_{2}^{3} x+\operatorname{ch}_{2}^{5} x\right) \operatorname{sh} \frac{1}{2} x}{\operatorname{sh} 3 x} .
\end{array}
$$

where $x \quad g \beta H_{\mathrm{E}} / 2 k T, H_{\mathrm{E}}$ is an effective exchange field, vide infra, $k$ is the Boltzmann constant and $T$ is the absolute temperature.

As one can see from Eq. (6), the crystal field gives no contribu tion to $e_{\mathrm{FeBO}_{3}}$.

With the spin Hamiltonian constants $D$ 0.1032, $a$

$0.0158, F \quad 0.0368 \mathrm{~cm}^{1}$ and $\alpha \quad 36^{\circ}$ specified recently [12], we obtain the following crystal field contributions to the magne tocrystalline anisotropy constants for $\mathrm{FeBO}_{3}$ at $0 \mathrm{~K}$ :

$$
\begin{array}{ll}
a_{\mathrm{cf}} & 4.82 \cdot 10^{5} \mathrm{Jm}^{3} \\
d_{\mathrm{cf}} & 2.55 \cdot 10^{3} \mathrm{Jm}^{3} . \\
e_{\mathrm{cf}} & 0
\end{array}
$$

\section{Dipole-dipole contribution to magnetocrystalline anisotropy}

\subsection{Model of a pair of fictitious magnetic charges}

Here we consider the interaction energy $\mathcal{E}$ between two paral lel/antiparallel identical dipoles for different models. Fig. 2 shows a system of two interacting dipoles implemented as a pair of

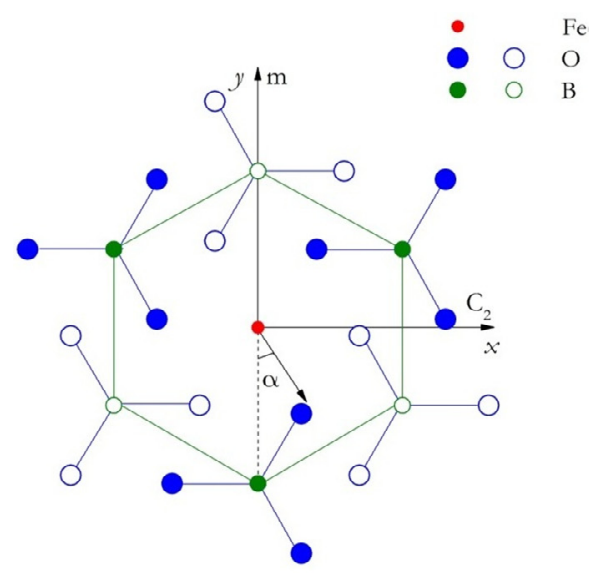

(a)

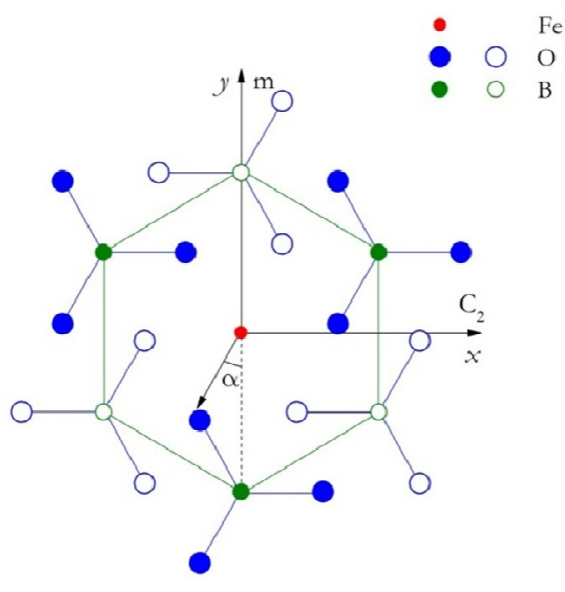

(b)

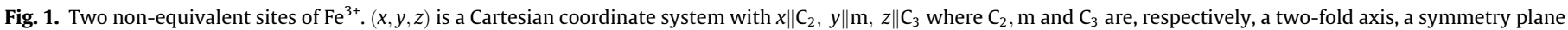

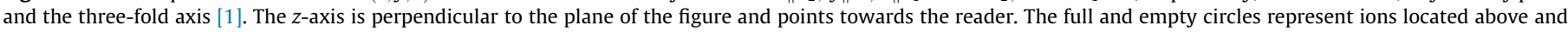
below this plane, respectively. 


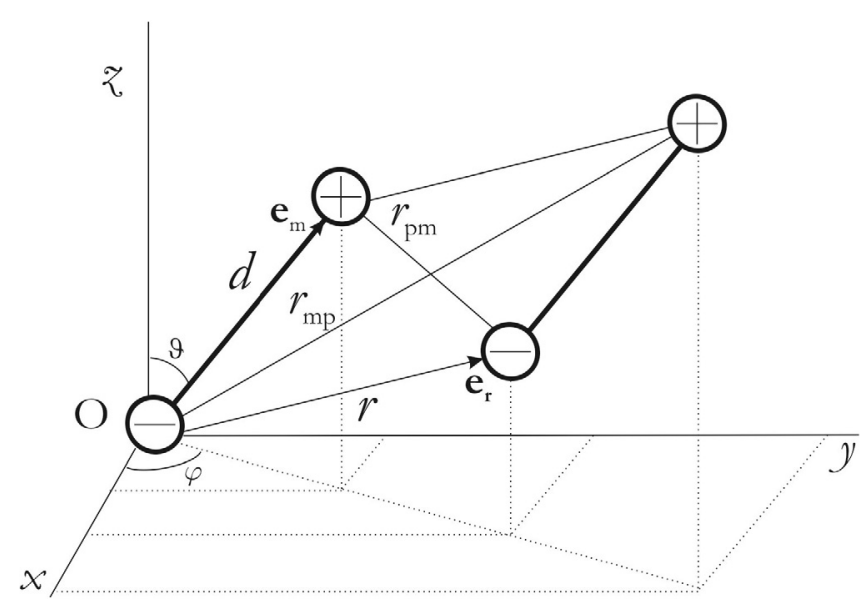

Fig. 2. System of two interacting dipoles in the model of a pair of magnetic charges.

"magnetic charges" $\pm q$ spaced a distance $d$ apart. For the dipole dipole energy one gets:

$\mathcal{E} \quad \pm \frac{\mu_{0}}{4 \pi} \frac{\mathrm{m}^{2}}{d^{2}}\left(\begin{array}{lll}\frac{2}{r} & \frac{1}{r_{\mathrm{mp}}} & \frac{1}{r_{\mathrm{pm}}}\end{array}\right)$

where $\mu_{0}$ is the permeability of vacuum, $m \quad q \boldsymbol{d} \quad q d \boldsymbol{e}_{\mathrm{m}}$ is the mag netic moment, defined by analogy with electrostatics and directed along the unit vector $\boldsymbol{e}_{\mathrm{m}}(\sin \vartheta \cos \varphi, \sin \vartheta \sin \varphi, \cos \vartheta)$; $r \quad\left(r_{x}^{2}+r_{y}^{2}+r_{z}^{2}\right)^{1 / 2}$ is the distance between the centers of the

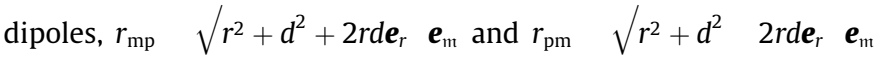
where $\mathbf{e}_{r}$ is the unit vector in the direction of $r$, see Fig. 2 for the notation. The choice of the \pm signs refers to parallel and antiparallel dipoles, respectively.

Introducing $\varepsilon \quad d / r$ in Eq. (9) one gets:

$$
\begin{aligned}
& \mathcal{E} \quad \pm \frac{\mu_{0}}{4 \pi} \\
& \left.\times \frac{\mathrm{m}^{2}}{d^{2} r} \quad 2 \frac{1}{\sqrt{ } 1+\varepsilon^{2}+2 \varepsilon \boldsymbol{e}_{r} \cdot \boldsymbol{e}_{\mathrm{m}}} \quad \frac{1}{\sqrt{ } 1+\varepsilon^{2} \quad 2 \varepsilon \boldsymbol{e}_{r} \cdot \boldsymbol{e}_{\mathrm{m}}}\right)
\end{aligned}
$$

For our purpose we need only an approximate expression of $\mathcal{E}$ for $\varepsilon \ll 1$. An expansion in the Taylor series up to the fourth order yields:

$\mathcal{E} \quad \mp 2 \frac{\mu_{0}}{4 \pi} \frac{\mathrm{m}^{2}}{r^{3}}\left(P_{2}+P_{4} \varepsilon^{2}+P_{6} \varepsilon^{4}\right)$

where $P_{n}$ are Legendre polynomials [14] of the scalar product $\boldsymbol{e}_{r} \boldsymbol{e}_{\mathrm{m}}$, and the and + signs correspond to parallel and antiparallel dipoles, respectively.

\subsection{Model of a circular current loop}

Next, we calculate the interaction energy between two identical and parallel circular current loops (Ampérian currents) of the same radii $R$ and areæ $S \quad \pi R^{2}$, carrying a current $I$, see Fig. 3. By defini tion, the magnetic moment of a loop is $m \quad S I \boldsymbol{e}_{\mathrm{m}} \quad \pi R^{2} I \boldsymbol{e}_{\mathrm{m}}$.

For definiteness, we choose the loops centered at the space ori gin $\mathrm{O}_{\mathrm{p}}$ and at an arbitrary point $\mathrm{O}_{\mathrm{s}}$ as the primary and secondary loops, respectively.

The dipole dipole interaction energy in this model is straight related to the mutual inductance $\mathcal{M}$ of the loops:

$\mathcal{E} \quad \mathcal{M} I^{2}$

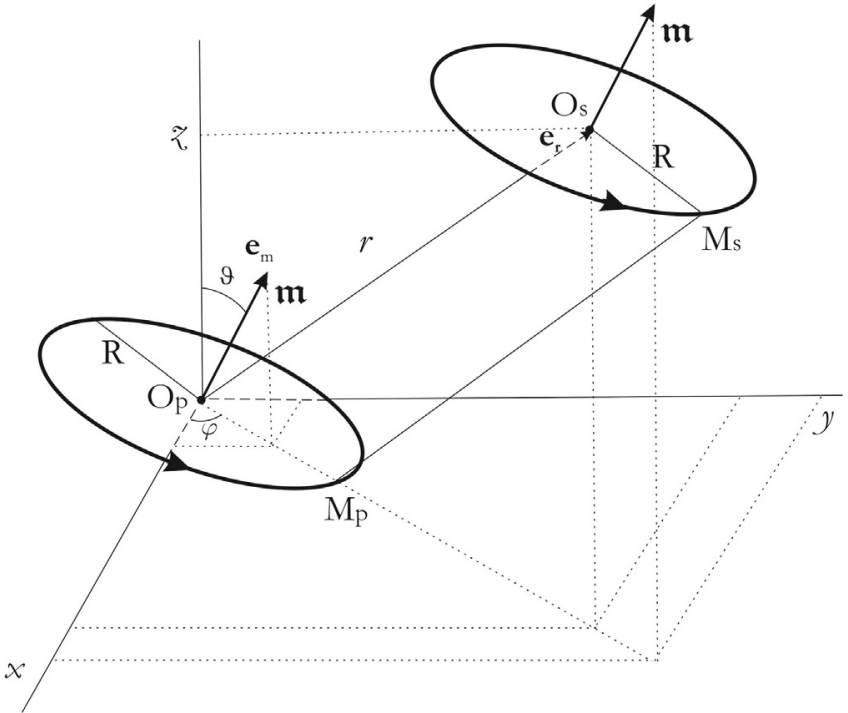

Fig. 3. System of two interacting Ampérian currents.

Here the sign in the right hand member occurs because the interaction energy between two coaxial (attracting) currents is negative while $\mathcal{M}$ in this case is positive. By definition,

$\mathcal{M} \frac{\Phi}{I}$

where $\Phi$, the magnetic flux induced by the current in the primary loop and passing through the secondary loop, can be calculated as follows:

$\Phi \oint_{l_{\mathrm{s}}} \oint_{l_{\mathrm{p}}} \mathrm{d} \boldsymbol{A} \cdot \mathrm{d} \boldsymbol{I}_{\mathrm{s}}$.

Here $l_{\mathrm{p}}$ and $l_{\mathrm{s}}$ are perimeters of the primary and secondary loops, $\mathrm{d} \boldsymbol{A}$ is a differential element of the vector potential at a point $M_{\mathrm{s}}$ on the secondary loop, produced by the primary loop:

$$
\mathrm{d} \boldsymbol{A} \quad \frac{\mu_{0}}{4 \pi} \frac{I}{M_{\mathrm{p}} M_{\mathrm{s}}} \mathrm{d} \boldsymbol{l}_{\mathrm{p}},
$$

$M_{\mathrm{p}} M_{\mathrm{s}}$ is the distance between two points on the primary and sec ondary loops, and $\mathrm{d} \boldsymbol{l}_{\mathrm{p}}$ and $\mathrm{d} \boldsymbol{l}_{\mathrm{s}}$ are differential elements of the corre sponding loops.

In order to evaluate the closed curve integrals in Eq. (14) we express $M_{\mathrm{p}} M_{\mathrm{s}}, \mathrm{d} \boldsymbol{l}_{\mathrm{p}}$ and $\mathrm{d} \boldsymbol{l}_{\mathrm{s}}$ as follows:

$$
\begin{aligned}
& \left|M_{p} M_{s}\right|^{2} \quad r^{2}+2 R^{2}\left[\begin{array}{lll}
1 & \sin (\phi & \tau
\end{array}\right] \\
& +2 R r_{x}[\sin \varphi(\cos \phi+\sin \tau)+\cos \varphi \cos \vartheta(\sin \phi \quad \cos \tau)] \\
& +2 R r_{y}[\sin \varphi \cos \vartheta(\sin \phi \quad \cos \tau) \quad \cos \varphi(\cos \phi+\sin \tau)] \\
& 2 R r_{z} \sin \vartheta(\sin \phi \quad \cos \tau)
\end{aligned}
$$

$\mathrm{d} \mathbf{l}_{\mathrm{p}} \quad\left(\begin{array}{l}R(\cos \varphi \cos \vartheta \sin \tau+\sin \varphi \cos \tau) \mathrm{d} \tau \\ R(\sin \varphi \cos \vartheta \sin \tau+\cos \varphi \cos \tau) \mathrm{d} \tau \\ R \sin \vartheta \sin \tau \mathrm{d} \tau\end{array}\right)$

and

$\mathrm{d} \boldsymbol{l}_{\mathrm{s}} \quad\left(\begin{array}{cc}R(\sin \varphi \sin \phi & \cos \vartheta \cos \varphi \cos \phi) \mathrm{d} \phi \\ R(\sin \varphi \cos \vartheta \cos \phi+\cos \varphi \sin \phi) \mathrm{d} \phi \\ R \sin \vartheta \cos \phi \mathrm{d} \phi\end{array}\right)$ 
In Eqs. (16) to (18) $\tau$ and $\phi$ are polar angles of arbitrary points of the primary and secondary loops, respectively. Putting these expressions in Eq. (14), for the interaction energy, cf. Eq. (12), we get:

$\mathcal{E} \quad \frac{\mu_{0}}{4 \pi} I^{2} \int_{0}^{2 \pi} \int_{0}^{2 \pi} \frac{\mathrm{d} \boldsymbol{l}_{\mathrm{p}} \cdot \mathrm{d} \boldsymbol{l}_{\mathrm{s}}}{M_{\mathrm{p}} M_{\mathrm{s}}}$

where the integrations are over $\tau$ and $\phi$.

Since, as in the previous case, we need only an approximate expression of $\mathcal{E}$, we can first expand the integrand in Eq. (19) in a Taylor series in the small parameter $\varepsilon \quad R / r$ and then integrate the result. In terms of the Legendre polynomials, up to the fourth order we get:

$\mathcal{E} \quad \mp 2 \frac{\mu_{0}}{4 \pi} \frac{\mathrm{m}^{2}}{r^{3}}\left[\begin{array}{ll}P_{2} & \left.3 P_{4} \varepsilon^{2}+\frac{75}{8} P_{6} \varepsilon^{4}\right]\end{array}\right.$

where the and + signs correspond to parallel and antiparallel dipoles, respectively.

\section{Calculation of the dipole-dipole interaction energy in $\mathrm{FeBO}_{3}$}

In order to calculate the dipole dipole contribution to the mag netocrystalline anisotropy constants for $\mathrm{FeBO}_{3}$, we have put for ward a computer code implementing the lattice sum method. We have chosen to do the summation in the volume of a rhombo hedron congruent to the primitive rhombohedron shown in Fig. 4.

The axes of the rhombohedral coordinate system $x^{\prime}, y^{\prime}, z^{\prime}$ coin cide with the edges of the rhombohedron, see Fig. 4. In transform ing the radius vector from the Cartesian to the rhombohedral system, we express the coordinates of iron sites through the edge length $l$ of the rhombohedron: $x^{\prime} \quad m l, y^{\prime} \quad n l$ and $z^{\prime} \quad k l$, where $m, n, k$ are integers numbering the sites along the corresponding axes. The radius vector in the new coordinate system is

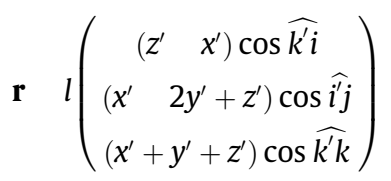

where $\cos \widehat{k^{\prime} i} \quad \sqrt{\frac{1 \cos \beta}{2}}, \cos \widehat{i^{\prime} j} \quad \sqrt{\frac{1 \cos \beta}{6}}$ and $\cos \widehat{k^{\prime} k} \quad \sqrt{\frac{1+2 \cos \beta}{3}}$ are the cosines of angles between the corresponding axes of two coor dinate systems. Substituting Eq. (21) in the expressions for the dipole dipole interaction energy, Eqs. (11) and (20) for the models of a pair of magnetic charges and a circular current loop, respec tively, we express this energy in terms of integers numbering the iron sites along the edges of the rhombohedron.

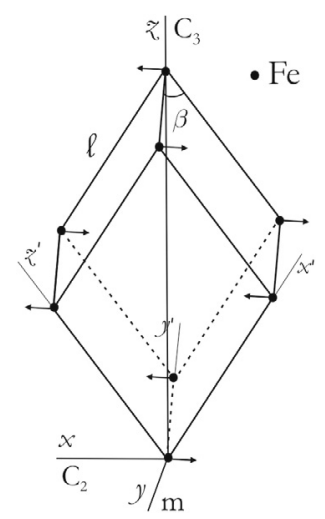

Fig. 4. A primitive rhombohedron with the edge length $l \quad 3.6 \AA$ and the apex angle $\beta \quad 79.9^{\circ}$ used to calculate the dipole-dipole energy.
The calculation of the dipole dipole energy density now is reduced to computing the following sum:

$\mathcal{E}_{\text {dip }} \quad \frac{1}{2} N \sum_{m, n, k}(1)^{m+n+k} \mathcal{E}(m, n, k)$

where the factor $(1)^{m+n+k}$ takes into account antiferromagnetic ordering and $\mathcal{E}(m, n, k)$ is the dipole dipole interaction energy between ions at the origin (numbered $0,0,0$ ) and at a site num bered $m, n, k$.

Henceforth, the magnetic dipole moment at $T 0 \mathrm{~K}$ will be expressed as

m $g \beta S$

where $g, \beta$ and $S$ have the same meanings as in Eq. (5).

The dipole dipole contributions at $0 \mathrm{~K}$, together with those of the crystal field, described in Section II, are listed in Table 1. One can see that the models of a pair of magnetic charges and a circular current loop result in substantially different expressions for the dipole dipole energies.

In order to get the dipole dipole contributions to the magne tocrystalline anisotropy constants at different temperatures, these contribution at $0 \mathrm{~K}$ should be multiplied by $\left(M_{T} / M_{0}\right)^{2}$, where $M_{T}$ is the sublattice magnetization at the temperature $T$. $\left(M_{T} / M_{0}\right)$ for $\mathrm{FeBO}_{3}$ have been tabulated [4]. The temperature dependences of the crystal field contributions are given in Eq. (6).

\section{Comparison with experiment}

From the AFMR experiments the effective basal magnetocrys talline anisotropy constant $e_{\text {eff }}^{\text {exp }}$, cf. Eq. (2), can be determined [15].

The EMR studies have been carried out at $77 \mathrm{~K}$ with a laboratory developed spectrometer at microwave frequencies $v$ from 15 to $36 \mathrm{GHz}$ and magnetizing field $H$ up to $10 \mathrm{kOe}$ applied in the basal plane of the crystal. $\mathrm{FeBO}_{3}$ crystal has been synthesized

Table 1

Crystal field and dipole-dipole contributions to the magnetocrystalline anisotropy constants of iron borate at $0 \mathrm{~K}$.

\begin{tabular}{|c|c|c|c|}
\hline $\begin{array}{l}\text { Constants, } \\
\mathrm{J} \mathrm{m}^{3}\end{array}$ & $\begin{array}{l}\text { Crystal field } \\
\text { contribution }\end{array}$ & $\begin{array}{l}\text { Dipole-dipole cont } \\
\text { Pair of fictitious } \\
\text { magnetic charges }\end{array}$ & $\begin{array}{l}\text { Circular current } \\
\text { loop }\end{array}$ \\
\hline$a_{\mathrm{FeBO}_{3}}$ & $4.82 \cdot 10^{5}$ & \multicolumn{2}{|c|}{$3.82 \cdot 10^{5}$} \\
\hline$d_{\mathrm{FeBO}_{3}}$ & $2.55 \cdot 10^{3}$ & $7.02 \cdot 10^{5} d^{2} / l^{2}$ & $-2.11 \cdot 10^{6} R^{2} / l^{2}$ \\
\hline$e_{\mathrm{FeBO}_{3}}$ & 0 & $-9.80 \cdot 10^{4} d^{4} / l^{4}$ & $-9.19 \cdot 10^{5} R^{4} / l^{4}$ \\
\hline
\end{tabular}

Table 2

Parameters in Eq. (24) and their experimental values for $\mathrm{FeBO}_{3}$.

\begin{tabular}{|c|c|c|}
\hline Parameter & Definition & Value at $77 \mathrm{~K}$ \\
\hline Dzyaloshinskii-Moriya field & $H_{\mathrm{D}} \quad \frac{1}{2}{\frac{D_{\mathrm{FeBO}}}{M_{T}}}^{\mathrm{a}}$ & $\begin{array}{l}99.3 \pm 0.2 \mathrm{kOe}[9] \\
98.68 \pm 0.52 \mathrm{kOe} \text { [this } \\
\text { work] }\end{array}$ \\
\hline Exchange field & $H_{\mathrm{E}} \quad \frac{1}{2} \frac{E_{\mathrm{FeBO}}}{M_{T}}$ & $\begin{array}{l}5.937 \cdot 10^{3} \mathrm{kOe} \\
6.02 \cdot 10^{3} \mathrm{kOe}^{\mathrm{b}}[5]\end{array}$ \\
\hline Isotropic energy gap & $H_{\Delta}^{2}$ & $\begin{array}{l}4.25 \pm 0.25 \mathrm{kOe}^{2}[9] \\
6.543 \pm 0.032 \mathrm{kOe}^{2} \text { [this } \\
\text { work] }\end{array}$ \\
\hline Anisotropic energy gap & $\underset{\mathrm{a}}{H_{\mathrm{E}} H_{\text {hex }}} \frac{1}{4} \frac{e_{\mathrm{eff}} E_{\mathrm{FeBO}}}{M_{T}^{2}}$ & $\begin{array}{l}0.05421 \pm 0.0013, \mathrm{kOe}^{2} \\
\text { [this work] }\end{array}$ \\
\hline $\begin{array}{l}\text { Basal magnetocrystalline } \\
\text { anisotropy field }\end{array}$ & $H_{\text {hex }} \quad \frac{1}{2}{\frac{e_{\text {eff }}}{M_{T}}}^{\text {a }}$ & $\begin{array}{l}-0.9 \cdot 10{ }^{5} \mathrm{kOe}[15] \\
0.913 \cdot 10{ }^{5} \mathrm{kOe}[\text { this } \\
\text { work] }\end{array}$ \\
\hline
\end{tabular}

a For $\mathrm{FeBO}_{3} M_{0} \quad 520 \mathrm{G}$ and $M_{77} \approx 512.9 \mathrm{G}$ [4].

b Value at $0 \mathrm{~K}$. 
by solution in the melt technique and had the shape of a thin hexagonal plate [6].

For $\boldsymbol{H}$ applied in the basal plane of the crystal, the low frequency AFMR mode for $\mathrm{FeBO}_{3}$ is described by the following expression $[15,16]$ :

$v \quad \gamma\left[H\left(H+H_{\mathrm{D}}\right)+36 H_{\mathrm{E}} H_{\mathrm{hex}} \cos 6 \varphi+H_{\Delta}^{2}\right]^{1 / 2}$

where $\gamma$ is the gyromagnetic ratio for $g \quad 2.0 ; H_{\mathrm{D}}$ and $H_{\mathrm{E}}, H_{\mathrm{E}} H_{\text {hex }}$ and $H_{\Delta}^{2}$ are, respectively, effective Dzyaloshinskii Moriya and exchange fields, anisotropic and isotropic energy gaps, $H_{\text {hex }}$ being the effective

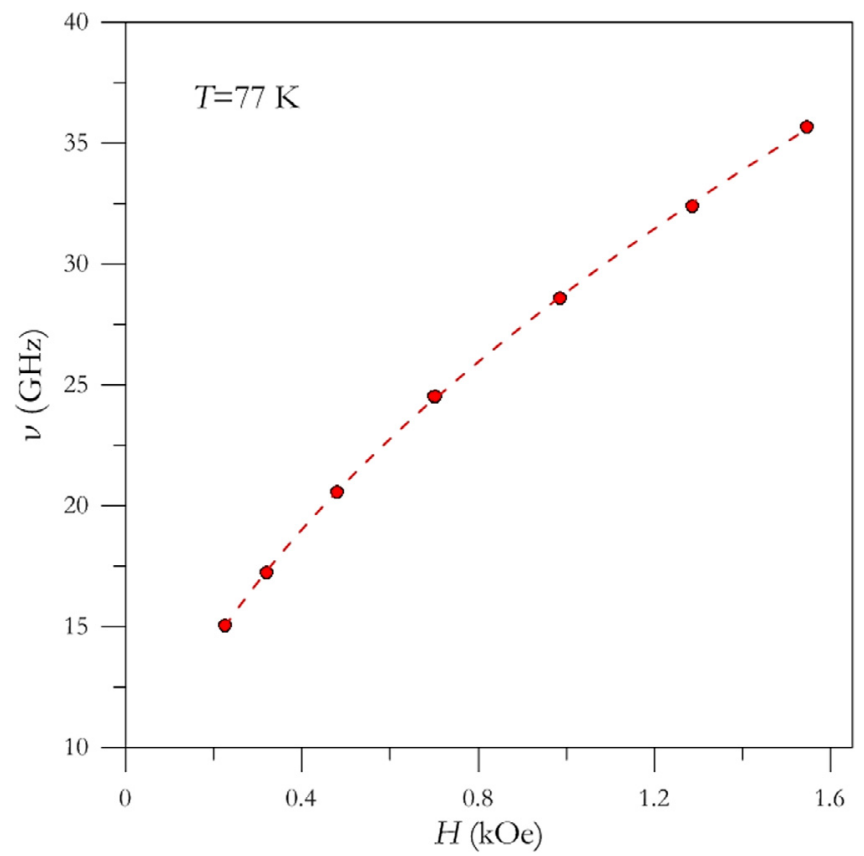

Fig. 5. Dependence of the AFMR frequency on the magnetizing field for $\mathrm{FeBO}_{3}$ crystal. The dashed curve is the best fit according to Eq. (24). field of basal magnetocrystalline anisotropy, and $\varphi$ is the angle between $\boldsymbol{H}$ and the $x$ axis. As far as different authors use different definitions of $H_{\mathrm{D}}, H_{\mathrm{E}}$ and $H_{\text {hex }}$, the definitions used in this work are given in Table 2 together with their experimental values.

The field sweep EMR spectra recorded at different $v$ allow obtaining the relation between $v$ and $H$. The experimental results shown in Fig. 5 can be convincingly fitted to Eq. (24), resulting in the following best fit parameters:

$$
\begin{array}{ll}
H_{\mathrm{D}} \quad & 98.68 \pm 0.52 \mathrm{kOe} \text { and } 36 H_{\mathrm{E}} H_{\text {hex }} \cos 6 \varphi+H_{\Delta}^{2} \\
& 6.46 \pm 0.34 \mathrm{kOe}^{2} .
\end{array}
$$

This value of $H_{\mathrm{D}}$ confirms the previous finding [9], see Table 2. Using this value, we have determined the product $H_{\mathrm{E}} H_{\text {hex }}$, see Eq. (24), from AFMR measurements at a fixed $v$, carried out by rotating $H$ in the basal plane of the crystal.

Fig. 6 shows the angular dependence of the quantity $(v / \gamma)^{2} \quad H\left(H+H_{\mathrm{D}}\right)$.

One can see that this dependence can be accounted for by a superposition of hexagonal and uniaxial magnetocrystalline aniso tropies in the basal plane. The occurrence of uniaxial magnetocrys talline anisotropy in this case can be due to a slight deviation of $\boldsymbol{H}$ from the basal plane or caused by mechanical stresses [17]. There fore, the angular dependence of the resonance field has been fitted to by the following expression, cf. Eq. (24):

$\left(\frac{v}{\gamma}\right)^{2} \quad H\left(H+H_{D}\right) \quad H_{\Delta}^{2}+36 H_{\mathrm{E}} H_{\mathrm{hex}} \cos 6 \varphi+p \cos 2 \varphi$

where $H_{\Delta}^{2}, H_{\mathrm{E}} H_{\text {hex }}$ and $p$ are fitting parameters, the term in $p$ describing the uniaxial component. The best fit parameters $H_{\Delta}^{2}$ and $H_{\mathrm{E}} H_{\text {hex }}$ are listed in Table 2 and $p \quad 1.13 \pm 0.05 \mathrm{kOe}^{2}$.

From $H_{\mathrm{E}} H_{\text {hex }}$ and $H_{\mathrm{E}}$ we get $H_{\text {hex }} \quad 0.913 \quad 10{ }^{5} \mathrm{kOe}$; then, using the definition of $H_{\text {hex }}$ given in Table 2, we get $e_{\text {eff }}^{e x p} 0.936 \mathrm{Jm}^{3}$. The $H_{\text {hex }}$ value corroborates that earlier reported by Doroshev et al. [15]; note that the negative sign of the latter value is due to a dif ferent definition of the angle $\varphi$.

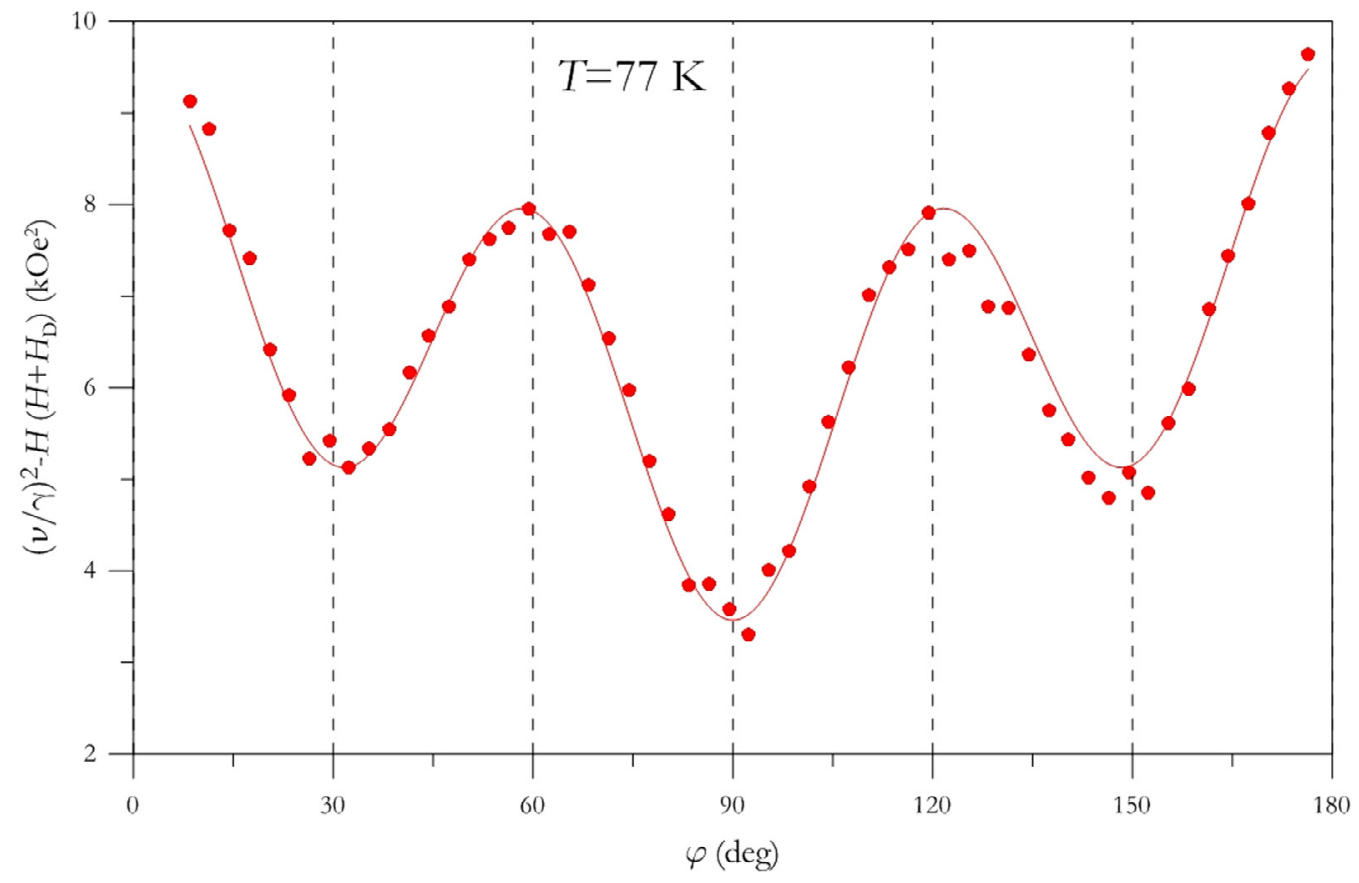

Fig. 6. Dependence of $\left(\frac{v}{\gamma}\right)^{2}-H\left(H+H_{\mathrm{D}}\right)$ on the angle $\varphi$. The curve is a fitting according to Eq. (25). 
Finally, from the results described above we estimate the dipole size. In the following we assume that $e_{\mathrm{eff}} e_{\mathrm{eff}}^{\mathrm{exp}}$, see Eq. (2) for $e_{\mathrm{eff}}$. Substituting $e_{\text {eff }}^{\exp }$ in Eq. (2) and taking into account Eqs. (3) and (4), we get:

$$
e_{\mathrm{eff}}^{\exp } e_{\mathrm{cf}}+e_{\mathrm{dip}}+\frac{1}{4} \frac{\left(d_{\mathrm{cf}}+d_{\mathrm{dip}}\right)^{2}}{a_{\mathrm{FeBO}_{3}}+\frac{D_{\mathrm{FeBO}}{ }^{2}}{E_{\mathrm{FeBO}}}} .
$$

In what follows, for $a_{\mathrm{FeBO}_{3}}$ we use the experimental value $a_{\mathrm{FeBO}_{3}}^{\exp } 3.210^{5} \mathrm{Jm}^{3}$ determined by AFMR at $77 \mathrm{~K}[4,9]$. $D_{\mathrm{FeBO}_{3}}$ and $E_{\mathrm{FeBO}_{3}}$ at $77 \mathrm{~K}$ have been calculated from the experimen tal values of corresponding effective fields and sublattice magneti zation, see Table 2. Substituting in Eq. (26) the abovementioned values as well as those given in Table 1 , for the model of a pair of fictitious magnetic charges we get:

$853 d^{4}+135 d^{2}+3.2 \quad 0.936$

Obviously, this equation can have only complex solutions; therefore, this model is not applicable in the actual case. In con trast, for the model of a circular current loop, with an analogous substitution, we get:

$$
7.47 \cdot 10^{3} R^{4} \quad 405.4 R^{2}+3.2 \quad 0.936,
$$

yielding two positive solutions: $R_{1} \quad 0.2189$ and $R_{2} \quad 0.0797 \AA$

In order to assess the plausibility of such values, they should be compared with the ionic radius $R_{\mathrm{i}}$ of $\mathrm{Fe}^{3+}$; indeed, we can reason ably infer that the effective size of a dipole should be of the same order of magnitude as the size of the physical object producing the corresponding dipole moment. For high spin $\mathrm{Fe}^{3+}$ in sixfold oxygen coordination $R_{\mathrm{i}} \quad 0.645 \AA$ [18]; therefore the $R_{1}$ value seems to be a more realistic estimate than $R_{2}$, the latter value being an order of magnitude smaller than $R_{\mathrm{i}}$.

The dipole dipole contributions to the magnetocrystalline ani sotropy constants of $\mathrm{FeBO}_{3}$ at $77 \mathrm{~K}$ calculated with the $R_{1}$ value are:

$e_{\text {dip }} \quad 12.2 \mathrm{Jm}^{3}$ and $d_{\mathrm{dip}} \quad 7.57 \cdot 10^{3} \mathrm{Jm}^{3}$.

\section{Conclusions}

Possible contributions to the basal magnetocrystalline aniso tropy of iron borate, namely, crystal field and dipole dipole contri butions have been considered in detail. The former contribution has been calculated using the spin Hamiltonian parameters for iso lated $\mathrm{Fe}^{3+}$ ions in (diamagnetic) gallium borate. The latter contribu tion has been evaluated assuming that the ratio dipole size/ interdipole distance is non negligible, i.e., that we are dealing with extended dipoles. The dipole dipole interaction energy has been calculated for two extended dipole models, viz., a pair of magnetic charges and a circular current loop. The dipole dipole contribution has been calculated by the lattice sum method.
In order to determine the basal magnetocrystalline anisotropy constants of iron borate, we have carried out AFMR studies at $77 \mathrm{~K}$. A comparison between the experimental and calculated val ues of this constant has shown that the model of a pair of magnetic charges fails at explaining the experimental results. In contrast, the model of circular current loop provides consistent evidence in sup port of the dipole dipole contribution to the basal magnetocrys talline anisotropy of iron borate and, incidentally, yields a more or less realistic estimate of the size of the magnetic dipole associ ated with $\mathrm{Fe}^{3+}$ ion.

In spite of the fact, that the modeling considering extended dipoles, put forward in this work provides new insight in the nat ure of the basal magnetocrystalline anisotropy of iron borate, it is certainly oversimplified. More sophisticated ( $a b$ initio) calculations are necessary in order to get further insight in the spatial distribu tion of the magnetic field produced by paramagnetic ions at short and intermediate distances.

\section{Acknowledgments}

This work was partially supported by the Russian Foundation for Basic Research and the Ministry of Education, Science and Youth of the Republic of Crimea in the framework of scientific pro ject Grant no. 1642910593 "p_a” and by the V.I. Vernadsky Crim ean Federal University Development Program for 20152024.

\section{References}

[1] R. Diehl, W. Jantz, B.I. Nolang, W. Wettling, in: E. Kaldis (Ed.), Current Topics in Materials Science, Elsevier, New-York, 1984, p. 241.

[2] M.B. Strugatsky, Thesis, Taurida National V. I. Vernadsky University, Simferopol, 2008.

[3] J. Kanamori, in: G.T. Rado, H. Suhl (Eds.), Magnetism, A Treatise on Modern Theory and Materials, Acad. Press, New York, 1963, p. 127.

[4] V.N. Seleznev, Thesis, Simferopol State University, 1988.

[5] V.G. Baryahtar, V.D. Doroshev, N.M. Kovtun, V.M. Siryuk, in: Abstracts of 19th Soviet Symp. on Low Temperature Physics, Minsk, 1976, p. 561.

[6] S. Yagupov, M. Strugatsky, K. Seleznyova, E. Maksimova, I. Nauhatsky, V. Yagupov, E. Milyukova, J. Kliava, Appl. Phys. A 121 (2015) 179.

[7] K. Seleznyova, N. Sergeev, M. Olszewski, P. Stepien, S. Yagupov, M. Strugatsky, J. Kliava, Solid State Nucl. Magn. Reson. 70 (2015) 38.

[8] V.V. Rudenko, V.N. Seleznev, A.S. Hlistov, in: Abstracts of Soviet Symp. on Physics of Magnetic Phenomena, Donetsk, 1977, p. 80.

[9] L.V. Velikov, A.S. Prokhorov, E.G. Rudashevskii, V.N. Seleznev, Sov. Phys. JETP 39 (1974) 909.

[10] K. Seleznyova, M. Strugatsky, J. Kliava, Eur. J. Phys. 37 (2016) 025203.

[11] S.N. Lukin, V.V. Rudenko, V.N. Seleznev, G.A. Tsintsadze, Sov. Phys. Solid State 22 (1980) 51

[12] K. Seleznyova, M. Strugatsky, S. Yagupov, N. Postivey, A. Artemenko, J. Kliava, Phys. Status Solidi B 251 (2014) 1393.

[13] S.A. Al'tshuler, B.M. Kozyrev, EPR in Compounds of Transition elements, 2nd Ed., Wiley \& Sons, New York-Toronto-Jerusalem-London, 1974.

[14] G. Korn, T. Korn, Mathematical Handbook for Scientists and Engineers, 2nd Ed. McGraw-Hill, New York, 1968.

[15] V.D. Doroshev, I.M. Krygin, S.N. Lukin, A.N. Molchanov, A.D. Prokhorov, V.V. Rudenko, V.N. Seleznev, JETP Lett. 29 (1979) 257.

[16] E.A. Turov, N.G. Guseinov, Sov. Phys. JETP 11 (1960) 955

[17] Yu.N. Mitsay, K.M. Skibinsky, M.B. Strugatsky, A.P. Korolyuk, V.V. Tarakanov, V. I. Khizhnyi, J. Magn. Magn. Mater. 219 (2000) 340.

[18] R.D. Shannon, Acta Crystallogr. A 32 (1976) 751. 